LEwin, R. A. (1954). J. gen. Microbiol. 11, 358-363.

\title{
Mutants of Chlamydomonas moewusii with Impaired Motility
}

\author{
By R. A. LEWIN \\ Maritime Regional Laboratory, National Research Council, Halifax, N.S., Canada \\ SUMMARY: A method is described for obtaining mutants of Chlamydomonas \\ moerousii with impaired motility. Thirteen distinct mutants with paralysed flagella \\ were isolated, and eleven of these were obtained in both mating types by crossing \\ with wild type. No sexual activity was elicited in M. 1060 (minus). No allelism was \\ detected among the remaining twelve mutants. In M. 1002 plus, the locus for \\ paralysis appears to be very closely linked with that of mating type. In certain \\ mutants, paralysed plus gametes, when paired with wild-type minus, recovered \\ motility after some hours. Recovery is attributed to the passage of motility factors \\ across the protoplasmic bridge linking the paired gametes. Paralysed minus cells \\ may act as donors of such factors when paired with paralysed plus cells of an unlike \\ mutant strain. The pairs formed by mating genetically dissimilar gametes are \\ considered as heterokaryons, which can be used to provide information on the \\ identity or non-identity of genes associated with paralysis. The role of a number of \\ transmissible factors in normal flagellar activity is thereby implicated.
}

In the protist flagellum as in muscle there must be some means by which chemical energy can be converted into movement, and it is likely that a chain of reactions may be involved. Genetic blockage or disturbance at any step will result in impaired motility, and from a study of genetically paralysed mutants we may hope to learn something of the processes controlling the normal functioning of flagella. Many unicellular algae or other flagellates lend themselves well to such studies. Paralysed mutants of Chlamydomonas moewusii and $C$. dysosmos have been obtained following ultraviolet irradiation: $C$. moerousii was selected for further study since the species is heterothallic, and thus more suitable for mating experiments and genetic analysis. Normal activity of wild-type cells has already been described (Lewin, 1952a).

\section{METHODS}

The culture medium contained, per litre: $\mathrm{Ca}\left(\mathrm{NO}_{3}\right)_{2} \cdot 4 \mathrm{H}_{2} \mathrm{O}, \mathbf{1} \cdot 0 \mathrm{~g} \cdot ; \mathrm{MgSO}_{4} \cdot 7 \mathrm{H}_{2} \mathrm{O}$, $0.2 \mathrm{~g}$.; $\mathrm{K}_{2} \mathrm{HPO}_{4}, 0.2 \mathrm{~g}$.; traces of $\mathrm{Fe}$, etc. (Lewin, 1952b) in glass-distilled water. Solid media were prepared with $1 \%$ Bacto-agar (Difco). When active gamete suspensions were required, the growth medium was supplemented with $1.0 \mathrm{~g} . / \mathrm{l}$. sodium acetate $\left(\mathrm{CH}_{3} \mathrm{COONa} .2 \mathrm{H}_{2} \mathrm{O}\right)$ and $1.0 \mathrm{~g} . / \mathrm{l}$. sodium citrate $\left(\mathrm{C}_{6} \mathrm{H}_{5} \mathrm{O}_{7} \mathrm{Na}_{3} \cdot 2 \mathrm{H}_{2} \mathrm{O}\right)$, and the procedure outlined by Lewin (1953) followed. All media were sterilized by autoclaving at $15 \mathrm{lb}$./sq.in. for $15 \mathrm{~min}$. Cultures were grown in constant light (220 f.c.) at controlled temperature $\left(23^{\circ}\right)$.

The origin of certain mutant stocks was described by Lewin (1952b). Others were obtained from plus or minus cells by the following enrichment technique. Cells in $15 \mathrm{ml}$. of suspension were irradiated with constant stirring for $15 \mathrm{~min}$. 
at a distance of $13 \mathrm{~cm}$. from a source of ultraviolet light (Westinghouse 'Sterilamp'). A number of $\mathbf{0 . 2} \mathrm{ml}$. samples was then removed and each inoculated into $10 \mathrm{ml}$. of mineral medium held in a $15 \mathrm{~cm}$. test tube, which also contained a $17 \mathrm{~cm}$. pipette, and which was covered by a glass vial. Tubes were illuminated for 7 days. The pipette was then used to remove cells from the bottom of each culture, in this way decreasing contamination with swimming cells from the upper layers. Samples so removed were streaked on agar plates and incubated in light for 10 days. Since colonies of wild-type cells tended to be more flattened than those of non-motile mutants, the more heaped colonies on each plate were selected, transferred individually to fresh liquid medium, and illuminated for a further 7 days. Mutant cultures could be distinguished from wild type by growth on the bottom of the tube rather than in the surface film. To eliminate duplication, only one clone was selected from each original enrichment tube. Among the mutants so obtained were palmelloid and flagella-less types (cf. Lewin, 1952b), which were rejected, as also were variants in which motility was only partially impaired.

To effect recombination between the mutant character and the mating-type locus, paralysed cells were mated by shaking in light with wild-type gametes, zygotes were isolated and germinated singly, and the resulting cell suspensions streaked on agar plates. Progeny clones arising from single colonies were tested for paralysis and mating type, and recombinants were selected, so that wherever possible both plus and minus clones were obtained for each mutant. Details of the genetic methods employed have been published elsewhere (Lewin, 1953).

\section{RESULTS}

\section{General characteristics of mutants}

All paralysed mutants conformed more or less to those already described (Lewin, 1952b). Swimming activity was impaired, so that cells in liquid cultures accumulated on the floor of the vessel (Pl. 1, fig. 1), but the ability to 'creep' (Lewin, 1952a) remained. Colonies on semisolid agar $(0.4 \%)$ media were thus capable of spreading, though at a rate considerably lower than that of wild-type (Pl. 1, fig. 2). In some mutants, cells capable of erratic swimming appeared in old cultures: in M. 1011 and M. 1038, rare spontaneous genetic reversions were isolated. Different strains showed small but constant differences in behaviour in cultures of comparable age and treatment. Cells of eight mutants were examined under the electron microscope, but in no case was the disability attributable to any visible structural change in the flagella (Lewin \& Meinhart, 1953). So far, all attempts to restore motility by the addition of various substances or cell extracts to the medium have been unsuccessful.

Since the flagella are intimately concerned with the processes of mating (Lewin, 1952a), it might be expected that certain paralysed mutants would also be impaired sexually. In fact, paralysed mutants in standing suspensions mated poorly; but the incidence of pairing was considerably improved when mixtures were shaken to ensure frequent flagellar contacts between cells of 
opposite mating type. M. 475 was exceptional in one respect: whereas the paralysed cells of both mating types paired fairly well with other paralysed mutants, virtually no pairing occurred when this mutant was selfed. Extracts of wild-type cells did not alleviate this self-sterility. M. 1060 (minus) appeared completely sterile, producing no pairs even when mixed with wild-type plus gametes.

Although M. 1002 plus produced sexually active gametes, all attempts to obtain recombination between this paralysis gene and mating type were unsuccessful. Fifteen zygotes were isolated from a cross (M. 1002 plus $\times$ wildtype minus), and from each zygote progeny-clones were tested for paralysis and mating type. In no case was a M. 1002 minus or wild-type plus strain isolated, indicating very close linkage between the M. 1002 gene and the mating-type locus.

\section{Behaviour of mutants in gamete pairs}

When wild-type cells are mated, copulating gametes form pairs which swim for a number of hours before cell fusion takes place, the cells being held together by a narrow bridge of protoplasm (Pl. 1, fig. 3) (Lewin, 1950). Only one gamete, the plus partner, is responsible for the propulsion of such pairs: the flagella of the minus cell cease to beat once the gamete protoplasts have become joined by a bridge, so that motility of the pair is unidirectional. When wildtype plus cells are paired with paralysed minus, the pairs are motile; whereas in the reciprocal cross the pairs fail to swim, since the plus partner is paralysed.

In well-illuminated cultures, cytogamy occurs after 4-8 hr., but in darkness it is delayed indefinitely. Pairs formed by the mating of wild-type minus gametes with plus cells of certain paralysed stocks, though initially nonmotile, began to swim after incubation for a period in darkness. It could be shown by suitable labelling, e.g. by the use of starved cells of one mating type (Lewin, 1950), that in such cases it was not the minus partner which had begun to swim, but the hitherto paralysed plus cell. Since recovery was not effected by culture filtrates from wild-type cells, it appeared that the plus mutant, paralysed as a result of some genetically controlled cytoplasmic deficiency, was able to receive the missing factor, essential for normal motility, from its non-paralysed minus partner. This is illustrated diagrammatically in Fig. 1.

Not all paralysed mutants proved capable of gaining motility when gametes were paired with wild-type cells; while, among those which were capable of recovery, the time required varied considerably. Recovery was more rapid at higher temperatures (e.g. $40^{\circ}$ ), but all cells lost motility within a few hours at this temperature. Recovery times given in Table 1 were determined for cells maintained at $20^{\circ}$ in darkness to suppress cytogamy.

Since the cytoplasts of the two gametes were connected by a protoplasmic bridge, we might regard such pairs as heterokaryons in which genetically unlike nuclei operate in a common cytoplasm. As in fungal heterokaryons, nuclei bearing mutant genes for different physiological impairments could complement one another to produce a wild-type phenocopy. Thus whereas pairs resulting from a selfing cross (e.g. M. $475 \times$ M. 475 , or M. $1001 \times$ M. 1001) were never observed to recover motility, the mating of certain dissimilar 


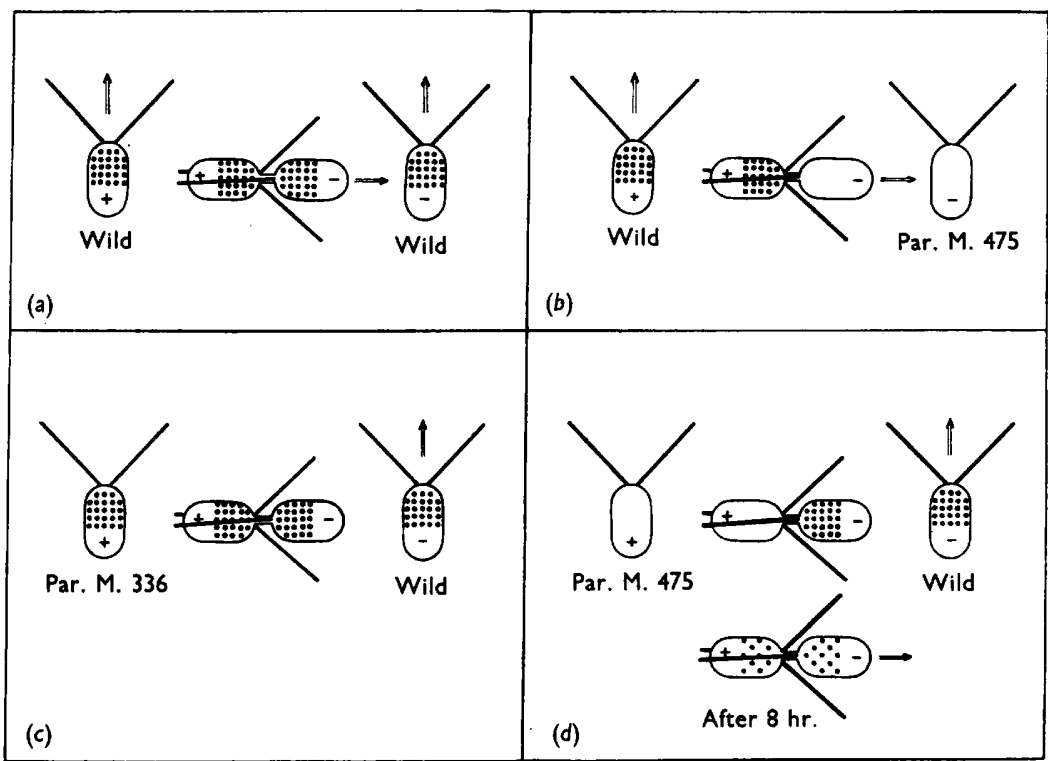

Fig. 1. Diagrams illustrating hypothesis of transmissible motility factors in Chlamydomonas moevusii. (a) Wild-type plus $\times$ wild-type minus. Pair motile as soon as gametes have copulated; propulsion by plus partner. (b) Wild-type plus $\times$ paralysed minus. Pair motile as soon as gametes have copulated. (c) Paralysed plus $\times$ wild-type minus. Pair remains immotile, since paralysis of M. 336 is not of 'recoverable' type. (d) Paralysed plus $\times$ wild-type minus. Pair initially immotile, since plus partner genetically paralysed, but becoming normally motile after incubation, after factor initially lacked by plus cell has been transmitted from wild-type minus partner. (Black spots represent motility factor. White flagella of M. 336 represent paralysis of non-recoverable type. Arrows indicate direction of swimming in motile cells or gamete pairs.)

Table 1. Times of recovery of plus paralysed mutants after pairing with minus wild-type gametes

\begin{tabular}{|c|c|}
\hline Mutant & Recovery time (hr.) \\
\hline M. 1002 & 2 to 4 \\
\hline $\left.\begin{array}{l}\text { M. } 475 \\
\text { M. } 1001\end{array}\right\}$ & 6 to 8 \\
\hline $\left.\begin{array}{l}\text { M. } 1017 \\
\text { M. } 1021 \\
\text { M. } 1022\end{array}\right\}$ & 24 to 48 \\
\hline $\left.\begin{array}{l}\text { M. 336* } \\
\text { M. 1011 } \\
\text { M. 1034 } \\
\text { M. 1038 } \\
\text { M. 1041 } \\
\text { M. 1043 }\end{array}\right\}$ & No recovery in 72 \\
\hline
\end{tabular}

* Grown with thiamine (1 mg./l.). 
mutants (e.g. M. 475 plus $\times$ M. 1001 minus, or M. 475 minus $\times$ M. 1001 plus) produced motile pairs when gamete mixtures, mated in light, were incubated in darkness.

An attempt was made to separate copulating gametes after the transfer of motility factors into hitherto paralysed cells. A mating suspension (M. 1002 plus $\times$ M. 1001 minus), containing abundant pairs, was incubated in darkness until the pairs, unlike the parental gametes, were actively motile. Samples taken after 3 and $24 \mathrm{hr}$. were subjected to forty up-and-down passages in a Potter-Elvehjem type homogenizer with a 'Teflon' pestle, resulting in the rupture of $40 \%$ of the pairs, and the organisms were then examined at intervals. Although a large proportion of the gamete pairs remained motile after such treatment, no motile unicells were observed, indicating either that separated plus ex-copulants reverted at once to their original paralysed state, or-more probably - that severe cell damage was an invariable concomitant of gamete separation.

Beadle \& Coonradt (1944) pointed out that indications of allelism might be obtained by the use of heterokaryon tests of this sort in fungi, though it should be borne in mind that proof of true non-allelism demands genetic analysis. Accordingly, twelve sexually active strains of paralysed Chlamydomonas moewusii, independently isolated after ultraviolet irradiation, and all but M. 1002 available in both mating types, were tested by pairing in all possible combinations. Portions of each gamete mixture were maintained in darkness for heterokaryon formation, and in light for zygote production. It was found that minus gametes of every mutant strain tested induced recovery of motility in plus gametes of 'recoverable' types (see Table 1), except in the cases of selfed pairings. Zygotes from each mating were allowed to germinate in liquid media, and, again in every case except selfings, clones of swimming cells could be isolated from the progeny, indicating that recombination had taken place between different paralysis genes.

\section{DISCUSSION}

In a previous study (Lewin, $1952 b$ ), seven paralysed mutants were obtained after ultraviolet irradiation, even without the use of a special enrichment technique such as that described here, and three of these mutant genes were tested and shown to be genetically non-allelic. It thus appeared that impairment of motility may be a common consequence of mutagenic irradiation, and that a number of different genes may be concerned in the processes of normal flagellar action. The results of the present paper bear this out, and indicate that at least a dozen genes, and probably many more, are involved. Certain of these genes are responsible for the formation of transmissible factors; the heterokaryon tests described above indicate that at least six different factors are implicated. Their nature is at present unknown. We may expect that their ultimate identification will be rendered difficult by the fact that they are apparently not liberated into the medium, and therefore presumably cannot pass freely through the cell membrane in either direction. 

Journal of General Microbiology, Vol. 11, No. 3

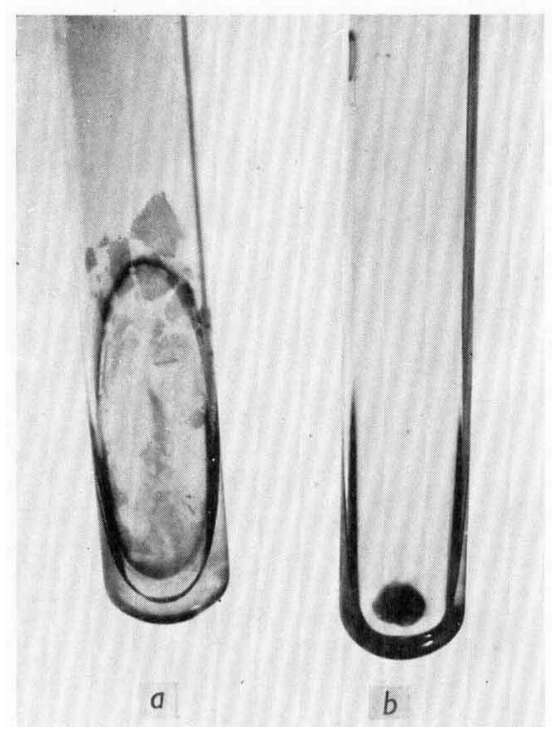

lig. 1

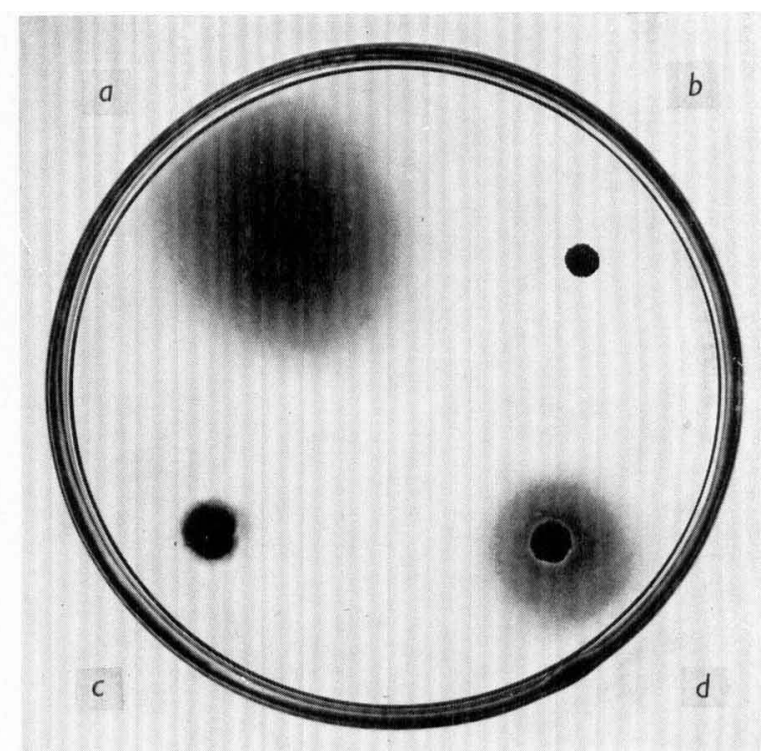

Fig. :

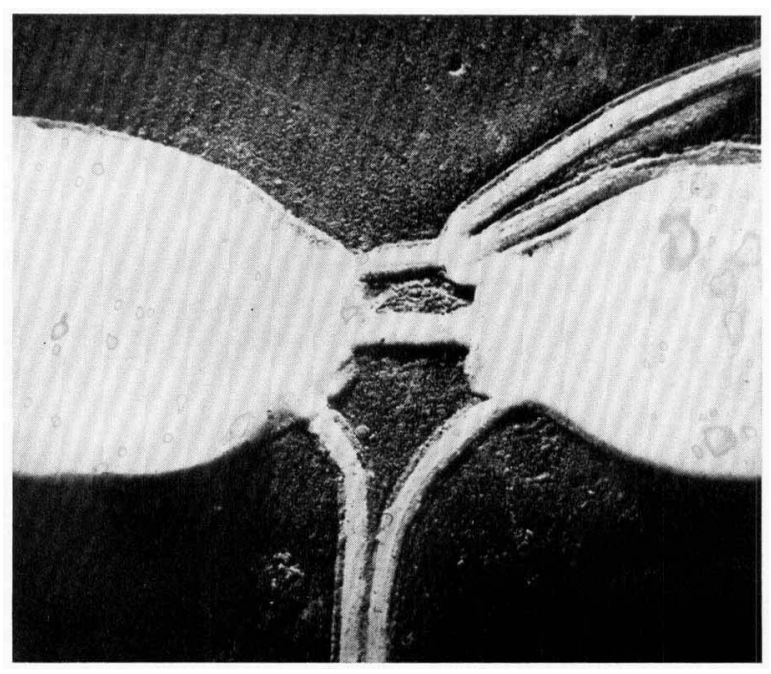

Fig. 3

R. A. LEWIN-C CHLAMYDOMONAS WITII IMPAIRED MOTLLITY 


\section{Chlamydomonas with impaired motility}

It should be emphasized that in these heterokaryon experiments with Chlamydomonas moewusii there was no evidence of genetic transduction, such as that demonstrated in Salmonella by Stocker, Zinder \& Lederberg (1953). Analyses of heterozygotes between wild-type and paralysed mutants yielded equal numbers of swimming and non-swimming clones, as expected according to currently accepted genetic theory.

This paper is the fourth in the series: Studies on the flagella of algae. It is issued as N.R.C. no. 1895.

\section{REFERENCES}

Beadle, G. W. \& Coonradt, V. L. (1944). Heterocaryons in Neurospora. Genetics, 29, 291.

Lewin, R. A. (1950). Gamete behaviour in Chlamydomonas. Nature, Lond. 166, 76.

LEwIN, R. A. (1952a). Studies on the flagella of algae. I. General observations on Chlamydomonas moewusii Gerloff. Biol. Bull., Woods Hole, 103, 74.

LEwIN, R. A. (1952b). Ultraviolet induced mutations in Chlamydomonas moewusii Gerloff. J. gen. Microbiol. 6, 233.

LEwin, R. A. (1953). The genetics of Chlamydomonas moereusii Gerloff. J. Genet. 51, 543.

Lewin, R. A. \& Meinhart, J. O. (1953). Studies on the flagella of algae. III. Electron micrographs of Chlamydomonas moewusii. Canad. J. Bot. 31, 711.

Stocker, B. A. D., ZinDER, N. D. \& Lederberg, J. (1953). Transduction of flagellar characters in Salmonella. J. gen. Microbiol. 9, 410.

\section{EXPLANATION OF PLATE}

Fig. 1. Sloped liquid cultures in test tubes. (a) Wild-type cells tend to accumulate in a surface film, which has here been disrupted by gentle agitation; (b) paralysed cells remain on the bottom.

Fig. 2. Spreading colonies on $0.4 \%$ agar, after 2 weeks growth. $(a)$ wild-type; (b) flagellaless mutant; ( $c$ and $d)$ paralysed mutants (M. 1017 and M. 475).

Fig. 3. Electron micrograph of gamete pair, showing bridge between anterior ends of copulating cells $(\times 10,000)$.

Photographs 1 and 2 by H. F. Roberts.

(Received 21 April 1954) 